\title{
Intereses y requisitos de la participación en organizaciones cooperativas cafetaleras costarricenses
}

Interesses e requisitos da participação em cooperativas de café da Costa Rica

Interests and requirements for participation in Costa Rican coffee cooperatives

- Doctora en Comunicación y Cultura por la Universidad Federal de Rio de Janeiro (UFRJ), Brasil.

- Profesora e investigadora de la Escuela de Comunicación (ECCC) en la Universidad de Costa Rica (UCR), Costa Rica.

- E-mail: lisbeth.arayajimenez@ucr.ac.cr 


\section{Resumen}

Al pensar en los requisitos e intereses que atraviesan el importante proceso de la participación de las personas asociadas a cooperativas, en este artículo se analiza la vigilancia y la confianza en diecisiete organizaciones del sector cooperativo cafetalero costarricense. Los datos muestran que existe confianza en la organización, identifican en el control democrático un área de mejora y destacan que la confianza constituye un requisito para que se dé la participación activa.

PALABRAS CLAVE: •COMUNICACIÓN ORGANIZACIONAL・PARTICIPACIÓN •VIGILANCIA •CONFIANZA・COOPERATIVISMO.

\section{Resumo}

Para pensarmos os requisitose interessesque intervêm no importante processo da participação dos cooperados, neste artigo analiso a vigilância e confiança em dezessete organizações cooperativas cafeeiras costarriquenhas. Os dados evidenciam a existência de confiança na organização, identificam no controle democrático uma área que tem de melhorar e ratificam a confiança como sendo um requisito para a participação ativa acontecer.

PALAVRAS-CHAVE: • COMUNICAÇÃO ORGANIZACIONAL • PARTICIPAÇÃO • VIGILÂNCIA •CONFIANÇA •COOPERATIVISMO.

\section{Abstract}

When thinking about the requirements and interests that permeate the important process of participation of cooperative members, this article analyzes the vigilance and trust in seventeen organizations of the Costa Rican coffee sector. Data indicate the existence of organizational trust, identifying an area that requires improvement regarding democratic control, and ratifying the role of trust as a requirement for active participation. 


\section{INTRODUCCIÓN}

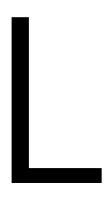

a relación entre comunicación y participación es estructural. Ampliamente conocidas son las líneas de trabajo epistémico, teórico, práctico y político desarrolladas en América Latina, las cuales hemos categorizado según sus especificidades bajo los nombres de comunicación popular, comunitaria y ciudadana, alternativa, e inclusive comunicación participativa. En esas perspectivas, la participación se entiende como medio y fin en sí misma, ya que procedió y creció de la mano de los movimientos sociales, populares, críticos de los modelos de "desarrollo" y de sus imposiciones colonialistas en el período entre 1960 y 1970.

Aun cuando no corresponde a estas líneas, pues en este artículo lo que se pretende es analizar intereses y requisitos de la participación en la vida organizacional; cabe únicamente referir, para las personas interesadas, la existencia de esas perspectivas y su importante papel tanto histórico como actual. A continuación mencionaremos algunos textos que nos permiten crear mapas cronológicos y traen discusiones y aportes al presente'.

Por su parte, en el campo que nos interesa, la importancia de la participación en la vida organizacional encuentra uno de sus referentes en los clásicos estudios de Elton Mayo, en los Estados Unidos, en la década de los años 1930. Casi un siglo después, los más diversos modelos de gestión organizacional han demostrado cuanto Mayo acertó.

En las organizaciones del así llamado "tercer sector" y de ellas en las cooperativas, podemos afirmar que la participación es vital², pues define la vida organizacional (Huertas, 2005, p.107). Como principio cooperativo garante la creación colectiva, el compartir los saberes y los haceres en un marco de respeto y compromiso con el bienestar colectivo.

Por ello, en nuestra área de trabajo y reflexión, la participación ha sido pensada de las más diversas maneras y ha tenido como foco diferentes actores de la vida organizacional; a veces los trabajadores, otras las/los asociados/as, en ocasiones los llamados públicos externos e inclusive los aliados estratégicos de la organización.

Un estudio acucioso de la participación debe considerarla un fenómeno complejo, por un lado porque se inscribe en dinámicas más amplias de procesos económicos, políticos, culturales y comunicacionales, tanto en los niveles macro como micro. Por otro lado, porque también se ve atravesada por las especificidades histórico-geográficas y sus relaciones de poder. Y finalmente, la participación es multicausal y multidimensional en atención a las dinámicas específicas que sus actores imprimen en el grupo humano concreto que se analiza, tanto en términos colectivos como subjetivos, en atención a las historias de vida de las personas que constituyen la organización.

Específicamente sobre el tema de la participación en cooperativas agrícolas, investigaciones previas ya habían comprobado la relación entre la permanencia del asociado y la sostenibilidad organizacional (Ciruela, 2006), por ello nos centramos aquí en el sector agrícola. La producción y exportación primero y su industrialización después dieron al café una relevancia histórica tanto para la economía y la política como para la cultura costarricense en general ${ }^{3}$. Las cooperativas cafetaleras,

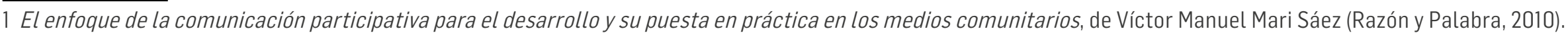

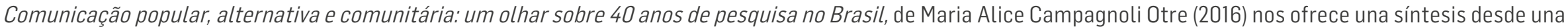

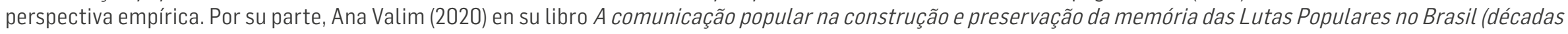
de 1970 e 1980) hace un recorrido en el que define cada una de esas formas de otra comunicación, bajo su interés de preservar la memoria histórica.

2 Lo mismo afirman Benetti y Baldissera (2018) para la comunicación en las organizaciones.

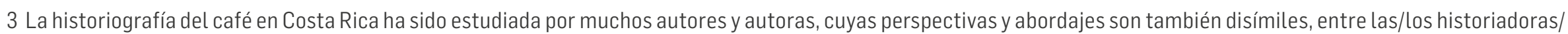

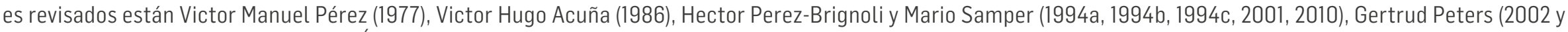
2004), Patricia Vega Jiménez (2004) y Álvaro Jiménez (2013). 
por su parte, tuvieron un papel fundamental al democratizar la producción y, especialmente, la industrialización del llamado "grano de oro", esa fue la motivación para investigar el sector cafetalero.

En los últimos años hemos observado como las plantaciones de este grano han cedido terreno a grandes condominios; haciendo eco de los procesos de gentrificación, como consecuencia del crecimiento urbano. La agricultura costarricense ha perdido el lugar primordial que tuviera otrora, agudizando los problemas de seguridad alimentaria al ser desplazada por los servicios, especialmente los servicios turísticos (que entraron en crisis con la pandemia por el covid-19).

El modelo cooperativo puede ser una salida estratégica a los problemas actuales, tanto aquellos que han surgido en las últimas décadas, producto de la implementación del capitalismo en su fase neoliberal más descarnada; relacionados con la catástrofe ambiental, la desigualdad socioeconómica, las crisis de empleo, subempleo e informalidad que enfrentan a enormes contingentes humanos con la sobrevivencia. Y también podríamos, a partir de este modelo organizativo, hacer frente a aquellas dificultades que son el resultado de la implantación de las lógicas de la empresa, del costo-beneficio y de la inversión, que pasaron a regir todos los ámbitos de la vida (Brown, 2015; Cooper, 2017), siendo el individualismo, el egoísmo, la competencia y el "cada uno por sí" las actitudes y comportamientos hegemónicos.

Con esas premisas teóricas y esas preocupaciones ciudadanas y profesionales en mente, en la tesis de la Maestría en Comunicación y Desarrollo de la que se desprende este artículo (documento completo localizado en el sitio del Instituto Nacional de Fomento Cooperativo -INFOCOOP4), se define una serie de dimensiones analíticas, entre ellas los roles y los ámbitos de la participación, sus grados, limitaciones, intereses y los requisitos para participar. En este artículo se aborda un componente de cada uno de los dos últimos, de los intereses la vigilancia y de los requisitos la confianza. La vigilancia entendida como una manera de ejercer control democrático de la organización, y la confianza comprendida como un requisito para la participación. La intención es colaborar desde la comunicación organizacional con uno de los desafíos que enfrenta el modelo cooperativo: hacer verbo su filosofía.

Para discutir los hallazgos, una versión preliminar de este texto se presentó al grupo Comunicación Organizacional y Relaciones Públicas, en el XIV Congreso de la Asociación Latinoamericana de Investigadores de la Comunicación (ALAIC), celebrado en San José, Costa Rica, en julio de $2018^{5}$.

Para este artículo, se amplió la discusión original apoyándose en autoras/es latinoamericanas/os, principalmente brasileñas (Arizaga, 2017; Benetti; Baldissera, 2018; Caldart, 1994; Henriques; Silva, 2018; Salgado, 2016; Silva; Baldissera, 2018; Silva, 2020) para pensar la participación, la confianza y la vigilancia, es decir, el poder, a partir de la comunicación organizacional. Además de las bases latinoamericanas, se incorporó en el debate conceptos de reconocidos sociólogos franceses, algunos de ellos dedicados hace décadas a pensar la contemporaneidad y a reflexionar sobre las maneras en que la gestión de lo humano en el trabajo se ha vuelto cada vez más insalubre (Bauman, 1998; Ehrenberg, 2017; Gaulejac, 2010).

\section{PRESUPUESTOS EPISTÉMICOS Y PRINCIPALES DIMENSIONES EN ANÁLISIS}

Esta investigación parte del presupuesto de que todo proceso organizacional está mediado por relaciones de poder y su distribución desigual entre los diversos actores. Además, las organizaciones son espacios conflictivos, de tensiones y complejidades, no uniformes, homogéneas, ni estáticas y sí contradictorias y en transformación.

4 Disponible en: https://www.infocoop.go.cr/index.php/publicaciones?keys=\&page=2

5 Disponible en: http://alaic2018.ucr.ac.cr/sites/default/files/2019-02/GT\%202\%20-\%20ALAIC\%202018_0.pdf. 
Para el caso de la vigilancia y con base en Ortega (2001 apud Huertas, 2005, p.106), se trabaja con la noción de participación como control entendida como la manera que tienen los asociados a cooperativas para ejercer (sea directa o indirectamente) la vigilancia organizacional.

Esta participación como control se relaciona, por tanto, con la vigilancia e incorpora una dimensión del poder inevitablemente adherida a la toma de decisiones. Adoración Mozas (2002, p.176) indica que esta "será efectiva si los miembros que participan en ella no solo detentan la responsabilidad, sino que además se sienten con verdadera fuerza para hacer uso del rol de tomador de decisiones en la sociedad cooperativa."

Bajo este mismo eje, Tjosvold (1995 apud Huertas, 2005, p.108) afirma que en espacios donde se da la cooperación las personas cederán con mayor facilidad sus cuotas de poder, porque el bienestar colectivo se prioriza, se coloca por encima de las metas particulares. Esa prioridad minimiza la competencia y permite que el interés central sea cumplir con los objetivos y fines organizacionales, pues alcanzar las metas de todos es alcanzar las metas individuales ${ }^{6}$.

Así las cuotas de poder, en un ambiente de cooperación, se delegan con más facilidad en aquellos miembros que el colectivo estima que sacarán la tarea. Esta idea difiere ampliamente del concepto de créditos de confianzaque utiliza Daniel Silva (2020, p.3, traducción propia), en su análisis de la influencia al pensar la relación de una organización con sus críticos, teniendo en última instancia "la intención de reflexionar sobre las lógicas presentes en las respuestas concretas que las organizaciones ofrecen delante de denuncias y acusaciones de abusos comunicativos".

En ese caso, como bien explica Silva (2020), la organización se vale de los créditos de confianza para que los públicos asuman que el discurso organizacional representa sus intereses, es decir, homologar el interés privado al público. Por el contrario, la participación, la cooperación y la solidaridad en las cooperativas son valores y principios, nortes filosóficos del quehacer organizacional; se parte de que el bien común está por encima del bien individual, así los fines organizacionales deberían responder al interés colectivo ${ }^{7}$. En ese escenario, confiar en aquellos con los que coopero y comparto intereses parece un acto racional.

Holtz (2004 apud Marchiori, 2011, p.50) argumenta que tal confianza se produce en la relación, en la interacción, siendo vincular, pues "la relación entre persona y persona y la interacción en tiempo real no puede ser sustituida por ninguna estrategia, por la confianza que se establece en esta relación".

La confianza está estrechamente asociada con la vulnerabilidad, pues supone que el asociado asuma que los otros (individualmente, en colectivo o en representación de la organización) no explotarán sus aspectos vulnerables (Morrow; Hansen; Pearson 2004 apud Huertas, 2005, p.108).

Precisamente, preocupado por esa vulnerabilidad, el comunicador brasileño Daniel Silva (2020) siguiendo la tónica que su equipo de investigación ha venido desarrollando se preocupa por los abusos comunicativos de algunas organizaciones privadas. Y se apropia de los desarrollos de León Mayhew, sociólogo estadounidense que analizó los procesos de influencia, quien a su vez revisitó los postulados teóricos originalmente formulados por Parsons a partir de la mirada de Habermas. Silva (2020) utiliza el concepto de créditos de confianza; por un lado, porque entiende que el volumen de información que

6 Reflexionando sobre la relación entre las jefaturas (gestores, mandos medios) y su papel como auscultadores, Silva y Baldissera (2018) usan el concepto de líder carismático, que sería aquel que, a partir de sus características personales, los subordinados pueden pensar que actuará en beneficio de todos en el grupo.

7 Por otro lado, el análisis aquí realizado se basa en la percepción de los asociados a las cooperativas y no en el discurso organizacional, por lo que no se pretende analizar las estrategias persuasivas de la organización, interés central de Silva (2020). 
se genera en la actualidad nos hace imposible su adecuada digestión y especialmente porque no tenemos el conocimiento necesario para opinar de manera informada sobre los más diversos temas.

Por ello, nos es imposible no confiar (estamos obligados a la confianza) y esto pone en evidencia el hecho de que dependemos de los otros. Si articulamos de manera resumida lo teorizado por las y los autores antes citados, podemos decir que confiar implica una relación de dependencia, que da cuenta de nuestra vulnerabilidad. Sin embargo, según la lectura que Daniel Silva hace de la propuesta de Mayhew, de su idea de créditos de confianza, no se trata de una confianza ciega ni total, y sí de un proceso de construcción y negociación, a través del cual las personas buscan señales o signos que les permitan creer, confiar; los llamados "indicativos de credibilidad" (Silva, 2020, p.8) que no siempre -y aquí nos atrevemos a afirmar, mucho menos hoy- son racionales.

Nos parece posible aseverar que lo que llamamos vulnerabilidad, al menos en una medida, se relaciona con la idea de incertidumbre que encontramos originalmente en Habermas y que Silva (2020) utiliza para su análisis basándose en Mayhew. Para Cecilia Arizaga (2017), es justamente la incertidumbre la emoción propia de nuestra época. Así, la confianza organizacional hoy enfrenta nuevos desafíos, pues ¿cómo confiar cuando vivimos en la era de la desinformación y las noticias falsas (fake news), la hiperexposición de la corrupción, la crisis de la democracia, la polarización de la sociedad, el descredito de la política y de la ciencia? Es en este contexto macro y micro donde procuramos identificar claves analíticas para interpretar la información colectada, partiendo del postulado de que todo dato es fruto y debe leerse a partir de las intrincadas tramas en que se produce.

\section{METODOLOGÍA}

Parece posible aseverar que la investigación en comunicación ha priorizado el enfoque cualitativo, por una parte, por la crítica histórica que con justa razón se ha hecho a la investigación cuantitativa por su cercanía con la línea funcionalista, promovida fuertemente desde los países anglosajones, y, por otra, porque en comunicación hemos enfatizado en la perspectiva simbólica. Podemos afirmar que como consecuencia de la reducida cantidad de estudios que utilizan el enfoque cuantitativo (comparativamente) se ha tendido al desarrollo rudimental de sus posibilidades y potencialidades, así como al desaprovechamiento de sus bondades.

Por lo anterior y con la intención de tener un mapa general del sector cooperativo cafetalero costarricense, en la investigación de la maestría de la que se desprende este artículo, defendida en noviembre del 2016, se empleó el enfoque cuantitativo, de tipo descriptivo-correlacional mediante el diseño transversal. Los datos fueron recolectados entre febrero y junio del 2015. Participaron en el estudio diecisiete cooperativas cafetaleras de Costa Rica, cuya extensión facilita los procesos de recolección de datos en la misma medida en que su topografía les impone restricciones.

Las 17 cooperativas aglutinaban una población total de 14.224 asociados. Para asegurar mayor representatividad y conociendo la diversidad que caracteriza el sector cooperativo costarricense en general y al cafetalero en particular, transformamos cada cooperativa en un estrato. A través de la selección sistemática (K o espaciamiento) y mediante la afijación proporcional, creamos una muestra probabilística de 306 personas, con un 96,6\% de nivel de confianza, un margen de error del 6\% y el nivel máximo de variabilidad. De las 306 personas elegidas al azar, 111 son mujeres (36,3\%) y 195 hombres (67,3\%); sin embargo, la variable sexo no se usó como referencia por lo que no representa las proporciones de su distribución en la población. Respondieron in situ (cara a cara) 203 personas, es decir, el 66,3\% de la muestra. A quienes definitivamente no fue posible localizar presencialmente se les aplicó la encuesta por teléfono, correspondiendo a 103 cooperativistas, el $33,7 \%$ de la muestra. 
La técnica de recolección de datos, el cuestionario utilizado ${ }^{8}$ fue revisado primero con funcionarios y gerencias de las cooperativas y del INFOCOOP; después, la prueba piloto se aplicó a personas de la población no seleccionadas en la muestra. A su vez la tabla de operacionalización de variables ${ }^{9}$ como corresponde a todo proceso de investigación riguroso hace posible identificar el proceso recorrido desde los conceptos hasta su medición en cada una de las preguntas del cuestionario. Los datos se procesaron en Statistical Package for the Social Sciences (SPSS). Para la descripción y análisis de los datos obtenidos, trabajamos con frecuencias simples y acumuladas, con cruces de variables (crosstabs) y construímos varios índices; en estas líneas describimos y comentamos los índices de vigilancia, de participación y de confianza.

\section{RESULTADOS Y DISCUSIÓN}

\section{El desafío de una participación sin control ni información}

La democracia es un valor, la participación es un principio en la filosofía cooperativa y su puesta en práctica un desafío. Por ello buscamos identificar la relación entre vigilancia y participación: "En la literatura sobre el mundo cooperativo, cuando se habla de participación, se refiere básicamente al principio de gestión democrática por parte de los socios" (Mozas, 2002, p.168).

Consultamos la percepción que tienen los asociados sobre el control de tres instancias fundamentales en la vida cooperativa, a saber, el Consejo de Administración, la Gerencia y el colectivo de personas asociadas (Asamblea). Según se muestra en el Gráfico 1 la mayoría (el porcentaje oscila entre 80-85\%) afirma no tener maneras de controlar las acciones de ninguna de dichas instancias. Apenas una sexta parte (11-15\%) asevera que tiene formas de ejercer la vigilancia.

Gráfico 1 - Vigilancia de las acciones de los diferentes órganos cooperativos

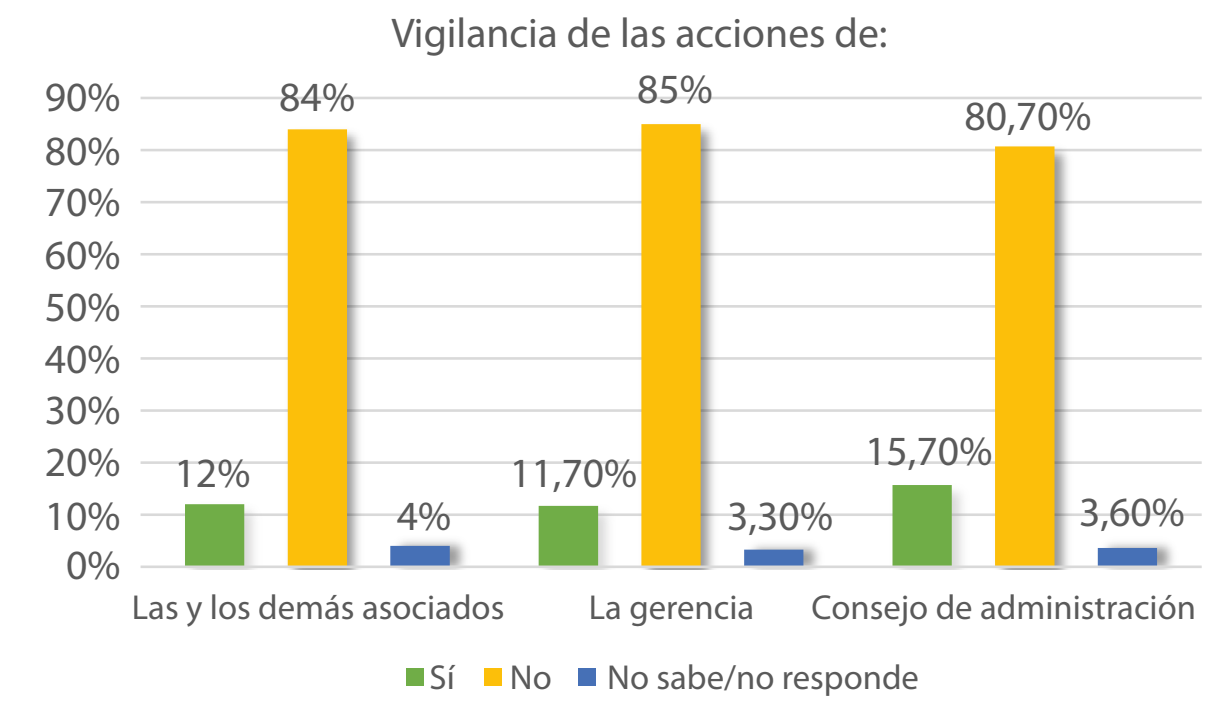

Fuente: Elaboración propia.

Construimos un índice de vigilancia que va de cero a tres ${ }^{10}$ (Tabla 1), según el cual cero (0) corresponde a los cooperativistas que afirmaron no tener ninguna forma de vigilar el accionar del Consejo de Administración, la Gerencia ni de la Asamblea de asociados, en los datos cero corresponde a la mayoría (76,8\%). Uno (1) representa control respecto de alguna de las tres instancias, apenas una décima parte así lo indicó (11,1\%). Dos (2) supone vigilancia sobre dos de los espacios de toma de decisiones y gestión de la organización, que solo el 7,5\% dice tener. Finalmente, el tres (3) representa en el índice el menor 
porcentaje ( $4,6 \%$ de las/los asociadas/os), se trata de quienes afirman contar con mecanismos de vigilancia para las tres instancias de la cooperativa. Nótese la tendencia decreciente.

Tabla 1 - Índice de vigilancia

\begin{tabular}{c|c|c}
\hline \multicolumn{3}{|c}{ Índice de vigilancia } \\
\hline & Absoluto & Porcentaje \\
\hline .00 & 235 & $76,8 \%$ \\
\hline 1.00 & 34 & $11,1 \%$ \\
\hline 2.00 & 23 & $7,5 \%$ \\
\hline 3.00 & 14 & $4,6 \%$ \\
\hline Total & 306 & $100,0 \%$ \\
\hline
\end{tabular}

Fuente: Elaboración propia.

En función de los datos y secundando a Ortega (2001 apud Huertas, 2005), nos parece posible aseverar que la participación como control no está siendo ejercida por la base asociativa cafetalera. Esto resulta desalentador si pensamos que en las cooperativas sus asociados son teóricamente los dueños de la organización, por ello su participación activa en el control de lo que se decide y hace es indispensable para la sobrevivencia organizacional, para cumplir con las metas y para cualquier cambio estratégico que se desee realizar en el camino.

La administración cooperativa establece una serie de dinámicas, procesos, estructuras y funcionamiento, desconocerlos limita seriamente la ejecución saludable de la gestión cooperativa. La investigación arroja luz sobre la dificultad de ejercer en la práctica una filosofía organizacional que se desconoce.

Por otra parte, un poco más de la mitad de la base asociativa $(55,5 \%)$ señala no tener maneras de saber si la información brindada en las asambleas es cierta, un tercio sabe que cuenta con mecanismos para comprobar dicha veracidad $(34,3 \%)$ y una décima parte (10,2\%) no sabe si existe o no una forma de verificación (Gráfico 2). Evidentemente existe una relación entre la verificabilidad de la veracidad de la información y la vigilancia del hacer organizativo.

Gráfico 2 - Verificabilidad de la veracidad de la información dada en la asamblea

Tiene manera de verificar si la información dada en la asamblea es verdadera:

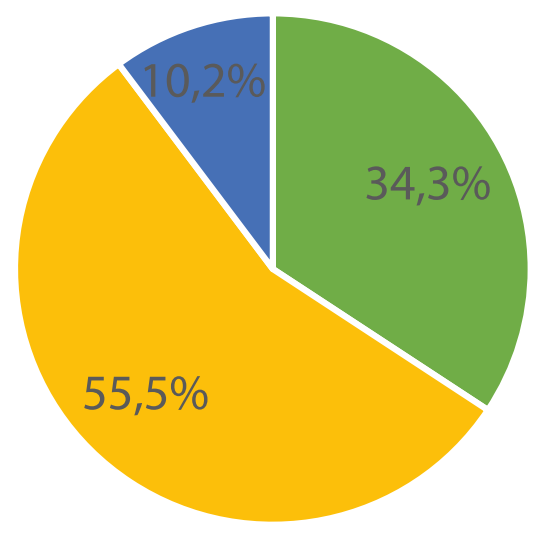

$$
\begin{aligned}
& \text { - Sí } \\
& \text { - No } \\
& \text { - No sabe/no } \\
& \text { responde }
\end{aligned}
$$

Fuente: Elaboración propia.

Esto resulta particularmente problemático en dos dimensiones, una vinculada directamente con la información como insumo para la toma de decisiones (en la acepción más funcionalista y clásica de la comunicación como información) y otra en su 
relación con el poder que, como sostiene Ignacio Martín-Baró (1999, p.99), "configura así el quehacer de personas y grupos [...] genera una realidad actualizada a través de ese quehacer: la realidad de lo que las personas mismas son."

Según la comprensión de participación democrática que se practica en los asentamientos y cooperativas del Movimiento de los Sin Tierra (MST) en Brasil, se da una falsa democracia en las asambleas en las que se busca tomar decisiones sobre cuestiones de las cuales la mayoría no está debidamente informada, pues seguramente privará la voz de quien convocó a la asamblea, "y eso es autoritarismo consentido" (Caldart, 1994, p.15, traducción propia).

Comulgamos con la idea de Henriques y Silva (2018) de que la construcción de credibilidad reviste una importancia notable y en ella el primer vector es la transparencia y el segundo cuanto la información es verificable. Podemos afirmar con base en los datos anteriores que las/los asociadas/os a cooperativas cafetaleras enfrentan serias limitaciones a su participación ${ }^{11}$, pues la mayoría reporta no tener formas de saber si la información que recibe en la asamblea es verdadera, al tiempo que dice tener poco o ningún control sobre las instancias de toma de decisiones. "Cuanto más se entiende lo que está sucediendo y quién está detrás de una iniciativa, mayor es la confianza en aquel proceso" (Henriques; Silva, 2018, p.68, traducción propia).

Recordemos que las asambleas de asociados son reuniones, normalmente anuales, en las cuales se presentan los informes de labores, es decir, se comunican los procesos y resultados tanto administrativos como financieros de la organización cooperativa. Las decisiones más importantes y estratégicas son tomadas en ese espacio, por ejemplo, el nombramiento de las personas en los puestos de dirección, la inversión de los excedentes y el rumbo de la organización para el siguiente lustro.

\section{Confianza como requisito para la participación}

Teóricamente defendemos la confianza como un requisito para la participación, por ello les preguntamos a los cooperativistas cafetaleros si las tres instancias, ya indicadas, son objeto de su confianza. Como muestra el Gráfico 3 dos tercios indica que, en efecto, los tres órganos la merecen. Debe llamar nuestra atención que porcentajes cercanos a dos quintos, en todos los casos, prefieran no confiar en los órganos de toma de decisiones organizacionales, así como el hecho de que (aunque con diferencias mínimas) confían más en el Consejo de Administración que en la Gerencia y menos en sus pares.

Gráfico 3 - Confianza según órgano corporativo
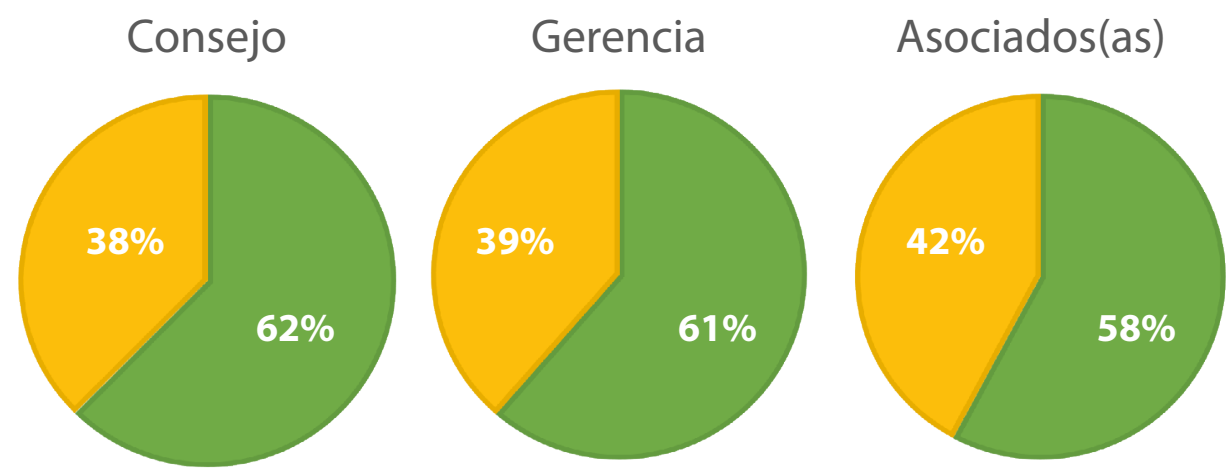

Puedo confiar

\section{Prefiero no contar}

Fuente: Elaboración propia.

\footnotetext{
11 En su análisis del papel de auscultadores desempeñado por las jefaturas en la comunicación con sus subordinados, Silva y Baldissera (2018) partiendo del estudio empírico de Silva (2016) evidenciaban que para la realidad brasileña, en los 12 casos estudiados en organizaciones de Rio Grande do Sul, la participación de los trabajadores subordinados ejercida en su real potencia en los procesos comunicacionales y de decisión constituía una práctica periférica. Por el contrario, dicha participación, que los autores definen como limitada, se reducía a: "a) decisiones restringidas a los flujos de cada equipo; b) contribuciones del empleado con ideas e innovaciones para la solución de problemas y para apoyar las decisiones de la jefatura; y c) manifestaciones que refuercen el punto de vista de la organización" (Silva; Baldissera, 2018, p.51, traducción propia).
} 
Para Samuel Garrido (2013) a partir de su experiencia empírica, es altamente más probable que la cooperativa funcione bien, en la medida en que exista mayor confianza entre los asociados, porque ella aumentará la disponibilidad de colaborar los unos con los otros. El autor acuña el concepto de costes de transacción y afirma que la confianza los haría disminuir; estos costes son aquellos en los que "incurren los agentes económicos (aunque solo sea invirtiendo un tiempo que podrían dedicar a otras actividades) con objeto de conseguir que los acuerdos (formales o de carácter informal) a que llegan con otros agentes sean respetados" (Garrido, 2013, p.22).

Él, que ha trabajado años con el sector agrícola sostiene que existe una relación entre respeto de las normas organizacionales y lo que llamamos paridad, así como entre cumplimiento de las normas y los fines organizacionales. Según esa comprensión, los asociados tenderán a respetar las normas establecidas en la cooperativa; si tienen seguridad de que los demás lo harán también (paridad) y si tienen además la certeza de que cumplirlas responde a los objetivos organizacionales. Por ello, Garrido (2013, p. 25) afirma que las personas tenemos con la cooperación una actitud de carácter condicional.

Si analizamos los resultados de la variable confianza según la óptica de Garrido (2013), es posible afirmar que la participación está ubicada en un escenario conflictivo, pues, aunque la mayoría confía, los porcentajes que no lo hacen alcanzan los dos quintos (cerca del 40\%); es presumible que esto esté teniendo impactos en la colaboración entre asociados.

Como muestra la Tabla 2, el índice de confianza igual que el índice de participación va de cero a tres. Se presentan los resultados de mayor a menor puntuación obtenida. Dos quintos señalan que las tres instancias son objeto de su confianza, una cuarta parte informa que solo dos de ellas y una quinta parte dice que ninguna -dato, sin duda, importante-; mientras que un sexto de los cooperativistas cafetaleros afirma que solo una de las tres instancias merece su confianza.

Buscamos saber si la variable género marcaba alguna diferencia en relación con la confianza. Como es sabido la mayoría de los asociados a cooperativas agroalimentarias, por razones culturales que aún avalan el machismo estructural, son hombres ${ }^{12}$. Aunque la diferencia obtenida no resulta estadísticamente significativa $(\mathrm{p}=.26)$, ellas son ligeramente menos confiadas que ellos (los promedios adquieren los valores de 1,67 para las mujeres y 1,89 para los hombres).

Tabla 2 - Índice de confianza

\begin{tabular}{c|c|c}
\hline \multicolumn{3}{|c}{ Índice de confianza } \\
\hline & Absoluto & Porcentaje \\
\hline .00 & 64 & $20,9 \%$ \\
\hline 1.00 & 46 & $15,0 \%$ \\
\hline 2.00 & 78 & $25,2 \%$ \\
\hline 3.00 & 118 & $38,6 \%$ \\
\hline Total & 306 & $100,0 \%$ \\
\hline
\end{tabular}

Fuente: Elaboración propia.

Pensemos ahora la relación entre confianza y vigilancia. Márcio Simeone Henriques y Daniel Silva han venido pensando en los últimos años la vigilancia como un tema relevante de la comunicación organizacional y pública (Silva; Henriques 2013; 2014; 2017; Henriques, 2015 apud Henriques; Silva, 2018). Especialmente en la medida en que sus características, potencialidades y desafíos quedan en relieve en relación con las posibilidades que permite el internet y los medios sociales

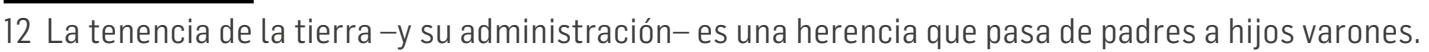


digitales, particularmente porque amplían la divulgación de informaciones y la colaboración (Henriques; Silva, 2018). Siendo este último otro esfuerzo analítico del Proyecto de Investigación llamado "Vigilância civil, opinião pública e accountability nas políticas urbanas" que es parte, a su vez, de las diversas iniciativas del Grupo de Investigación Dialorg.

Henriques (2015, p.207) apunta que la vigilancia civil puede ser entendida como una práctica de públicos más o menos organizados que buscan observar actividades de instituciones públicas y privadas y los procesos de comunicación pública, apuntando para "sus posibles consecuencias y las formas en que afectan a los individuos y a la sociedad" (Henriques; Silva, 2018, p.59, traducción propia).

Como vimos, la mayoría (60\%) de los asociados a las cooperativas confían en sus instancias de representación. Si sumamos los datos del índice de confianza, para las instancias en que los cooperativistas confían obtenemos una abrumadora mayoría (80\%). Los postulados de Henriques y Silva (2018) abordan la relación entre desconfianza y vigilancia ${ }^{13}$, indicando que la segunda se da, al menos en una medida, porque se desconfía de las informaciones y las fuentes oficiales. Si invertimos ese presupuesto en este análisis y agregamos la idea de costes de transacción de Garrido (2013) antes explicada, podríamos pensar que las/los asociadas/os a cooperativas cafetaleras costarricenses pueden no estar participando, justamente porque la confianza puntúa alto. Es decir ¿para qué me voy a involucrar si confío en quienes toman decisiones y me representan? Al profundizar en los datos mediante las correlaciones y su significancia, desmentimos esa potencial interpretación.

Con el objetivo de profundizar en la participación, se buscó conocer cuánto y cómo asisten los asociados a las asambleas, para ello se utilizó una tipología de participación que incluye no participación (no asiste a las asambleas), participación pasiva (asiste y únicamente vota) y participación activa (asiste, tiene voz y voto).

Al cotejar el promedio de la confianza con los tipos de participación identificamos que la distancia entre no participar y hacerlo pasivamente no es significativa, es decir, no ir a las asambleas o asistir para votar-únicamente-no guarda distancia en relación con la confianza.

Como muestra el Gráfico 4, el dato relevante aparece en relación con lo que llamamos aquí "participación activa" (es decir, intervenir en las asambleas proponiendo ideas e intentando inclusive convencer a los asambleístas), la cual encuentra en la confianza un requisito. Antonio Ciruela trabajó en el 2006 con la misma cantidad de cooperativas cafetaleras costarricenses y en su análisis ya había identificado esa correlación estadísticamente significativa al afirmar que, cuanta mayor confianza se tiene, más se participa. La presente investigación confirma, actualiza y amplía esa relación.

Los estudios de otro analista de organizaciones cooperativas agrícolas ya citado aportan la variable tiempo para fortalecer esta interpretación. Según Garrido (2013, p.23), existen diferencias en los resultados de la cooperación en función de los plazos -corto, mediano o largo-. Garrido denomina trampa social al hecho de que, en función de la desconfianza, se toman decisiones en el tiempo.

En el análisis de la aplicación "Tá faltando água" que procura crear datos independientes para georreferenciar los problemas de abastecimiento de agua en la ciudad de São Paulo, en Brasil; Henriques y Silva (2018) señalan indirectamente la variable tiempo al reconocer la diferencia que marca la utilidad inmediata de la participación. Al comparar la aplicación mencionada con el Waze, indican que esta última genera una recompensa inmediata para la vida de las personas, que no tiene el caso de su estudio; lo cual, claro, no disminuye el impacto político de los datos generados por dicha aplicación. Los autores evidencian una relación de tensión entre las dimensiones política y temporal; siendo que la utilidad inmediata genera involucramiento.

13 Traen a su discusión los interesantes conceptos de democracia de la desconfianza y contra-democracia formulados por Pierre Rosanvallon. 
Gráfico 4 - Tipo de participación en asambleas según índice de confianza

\section{4}

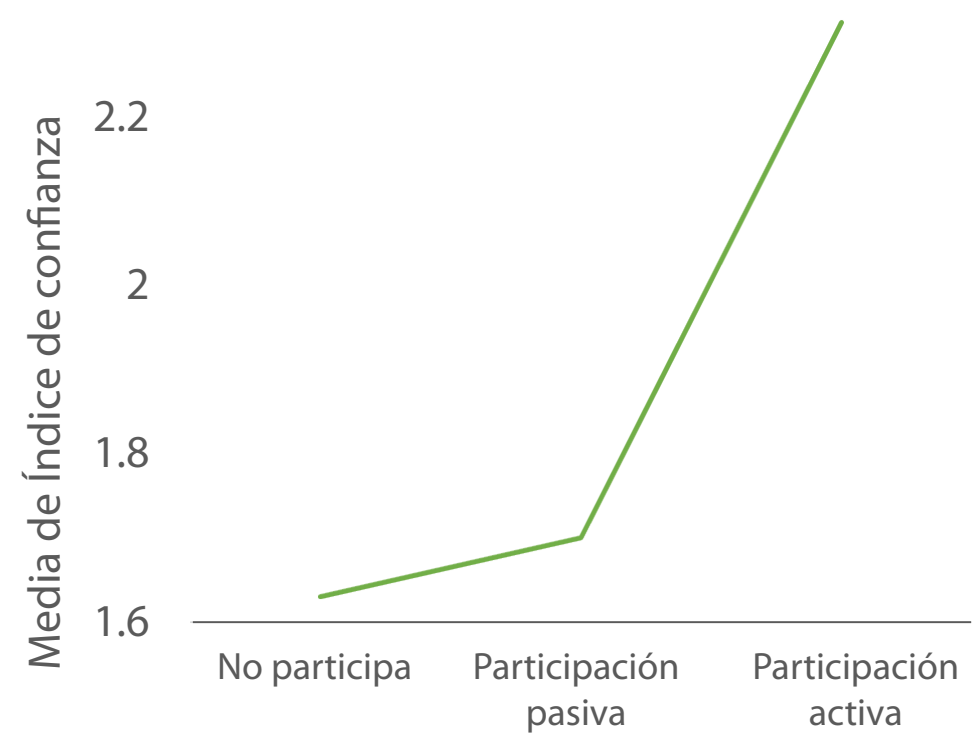

Tipos de Participación

Fuente: Elaboración propia.

Reconocemos así la variable tiempo como un factor que considerar cuando de participación se trata. El tiempo en general y, más puntualmente, la inmediatez, especialmente en nuestros contextos, como bien dice Júlia Salgado (2016), hacen que nuestra sociedad sea indispuesta con la demora.

La mayor parte de los asociados, aún sabiendo que en el largo plazo recibirán más beneficios para el colectivo si cooperan, eligen accionar en el corto plazo donde los beneficios son mayores si no cooperan como consecuencia de la desconfianza. En el mediano plazo, adiciona el autor, la desconfianza tiene otras consecuencias desafortunadas, porque genera un clima de enfrentamiento entre las personas que conforman la base asociativa. Finalmente, el teórico asegura que en el largo plazo la desconfianza supera las experiencias concretas de una generación instalándose en el imaginario colectivo, especialmente cuando se recuerdan las heridas que sufrieron los antecesores, y sin necesidad incluso de conocer lo que sucedió, las nuevas generaciones rechazan el modelo por causa de la desconfianza y sus efectos (Garrido, 2013, p.26).

Parece racional interpretar que la desconfianza expresada por los cafetaleros, sean cuales fueren las circunstancias que la causaron, puede estar afectando negativamente su interés por los bienes, procesos, decisiones e intereses colectivos; disminuyendo su implicación y su función vigilante de la marcha organizacional.

A partir de estos presupuestos conceptuales y epistemológicos, analizamos la información recolectada, especialmente aquella que ofrece luz sobre la relación entre confianza y participación activa, y afirmamos que la escaza participación activa y la apuesta por decisiones y acciones de corto plazo tiene, al menos en una medida, correlato con los hechos históricos que han generado desconfianza, sea en general en los modelos asociativos (como evidenció Garrido, 2013), sea específicamente sobre el accionar de los órganos que conforman las cooperativas.

Martín-Baró (1999) coincide con Garrido en relación a la importancia de mantener los fines organizacionales, aunque su lectura no enfatiza en la participación o la confianza y sí en la identidad y la pertenencia. Sin embargo, extrapolaremos el análisis organizacional del teórico salvadoreño para mirar la confianza y sus fisuras. Para el autor, cuya perspectiva política tuvo y tiene gran influencia en la psicología social centroamericana, la naturaleza de la organización, del grupo social organizado, puede cambiar si este olvida su finalidad, la razón de su existencia. Martín-Baró afirma inclusive que tal olvido puede conducir a la extinción de la organización. Bajo estos postulados nos preguntamos no con poca preocupación ¿será 
que las organizaciones cooperativas, siguiendo la percepción de los cooperativistas, podrían llegar a perder su finalidad si la confianza sigue disminuyendo y también lo hace la participación activa?

Como afirman varios/as autores/as, especialmente a partir de la sociología francesa, la contemporaneidad se caracteriza por la búsqueda permanente de nuevas emociones (Ehrenberg, 2017; Gaulejac, 2010), que responde a una identidad en construcción incesante donde gobiernan la desconfianza y la incertidumbre (Arizaga, 2017; Bauman, 1998). Esta última autora -socióloga argentina-asevera inclusive que el nuestro es "un mundo que se ha vuelto incierto y hostil ante nuevas formas de flexibilidad, cambio y riesgo planteadas por el capitalismo y que desde el mundo del trabajo se expanden a todas las áreas de vida de las personas" (Arizaga, 2017, p.14). Costa Rica no escapa a esa realidad.

Entendiendo que constituye un error metodológico (referido específicamente a la interpretación) sacar los datos de contexto 0 no regresarlos a él; en las siguientes líneas y de manera muy breve, intentamos mirar la información que nos han aportado los asociados a organizaciones cooperativas cafetaleras costarricenses, con el lente más amplio del entorno nacional. Afirmando que las dimensiones de la participación que se analizan (confianza y vigilancia) no responden únicamente a las subjetividades particulares y características organizativas, sino que están inseridas en un contexto de incertidumbre y desesperanza que describíamos anteriormente, donde el individuo reina soberano y la competencia, más ampliamente la lógica de la empresa, abandonó el ámbito específico del mercado para instalarse como norma de comportamiento general (Salgado, 2016), según la cual el compromiso primero y prioritario es con la propia agenda y el privado interés.

El Índice de Confianza del Consumidor (ICC) es una manera de identificar los niveles de optimismo (valores del índice cercanos a 100), pesimismo (valores cercanos a 0); mientras que calificaciones que ronden el 50 representan la incertidumbre y la neutralidad (Madrigal-Pana, 2014, p.3). Dicha medición se realiza trimestralmente. La encuesta de mayo del 2020 muestra que la confianza de los consumidores es de 36,6 puntos, lo cual no dista de lo obtenido en febrero del presente año (es decir, con antecedencia a la aparición del coronavirus), medición que refleja incertidumbre y pesimismo14.

Pero estos datos no son novedosos, si vamos atrás en el tiempo hasta los gobiernos de Laura Chinchilla (2010-2014), Oscar Arias (2006-2010) y Abel Pacheco (2002-2006), encontramos que el ICC fue mejorando con los años (pasando de 43,4; a 44,9; a 39,4); sin embargo, en ningún caso alcanzó el optimismo; muy por el contrario, los valores se mantuvieron siempre bajo la línea de neutralidad e incertidumbre que representan los 50 puntos.

Con ese pesimismo costarricense colaboran -como en otras partes del mundo- los hechos de corrupción privada y pública que han ocupado las primeras planas de los medios masivos del globo; y las fluctuaciones en la economía local producidas y acentuadas por las crisis internacionales. A su vez los movimientos sociales y políticos con sus reivindicaciones han puesto en evidencia una amplia gama de problemáticas (sea de acceso al agua, a la tierra, a la salud, a la educación, o a la vida misma).

Problemáticas estas que se acentúan actualmente con el resurgimiento de los conservadurismos y especialmente en atención a la aplicación cada vez más descarnada del capitalismo y su necropolítica (Mbembe, 2018), impuestas en Costa Rica en los últimos 40 años, cuyos indicadores más radicales son la desigualdad y la pobreza. Según la Encuesta Continua de Hogares del Instituto Nacional de Estadística y Censos de Costa Rica (INEC) del 2019, durante los últimos 10 años, el coeficiente GINI ha alcanzado más de 0,5 puntos, ubicándose en el 2019 en 0,508, para ese mismo año, la pobreza llegó al 20,98\% de los hogares, con un $5,84 \%$ de ellos en pobreza extrema (INEC, 2019).

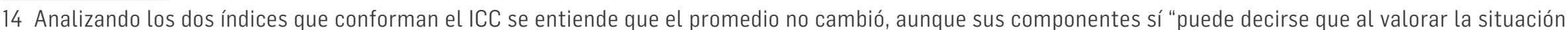

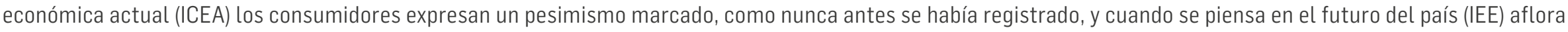
el optimismo" Madrigal-Pana (2020, p. 4)
} 
Todos esos antecedentes son caldo de cultivo en el que se inserta la desconfianza de las/los asociadas/os a cooperativas de café, siendo dicha desconfianza un aspecto mucho más amplio de la incertidumbre política y económica en las complejas dinámicas del contexto local, regional e internacional; y no únicamente una dimensión organizacional y mucho menos una característica exclusivamente subjetiva.

\section{CONSIDERACIONES FINALES}

Con base en los procedimientos estadísticos utilizados para analizar la vigilancia, las cuotas de poder con las que dicen contar los asociados a las cooperativas cafetaleras costarricenses contradicen las posibilidades de acercar la filosofía a la práctica cooperativa, especialmente en lo relativo al control organizacional. En coherencia con una participación más bien limitada (pasiva o nula), no se está ejerciendo la denominada por Ortega (2001 apud Huertas, 2005) participación como control. Parece necesaria una reflexión sobre la dimensión política que atraviesa esa ausencia de participación vigilante, en la medida en que retrata los niveles de empoderamiento de la base asociativa en relación con sus organizaciones.

Parece que esta problemática no ha sido atendida con seriedad, aunque ya el XIII Congreso Nacional Cooperativo (2014, p.39) indicó que la capacitación estaba siendo insuficiente y necesitaba reforzarse el empoderamiento de los socios, justamente en relación con su control de la organización. Son deseables nuevas investigaciones en esta materia para definir si se implementaron estrategias de comunicación o capacitaciones y evaluar su apropiación y puesta en práctica. 0 por el contrario para definir cuáles son las ganancias secundarias de no atender directamente las falencias en la participación de las/los asociadas/os.

El análisis de la participación no puede dar por sentadas las relaciones de poder, puede analizarlas en los espacios específicos de interacción en los que adquieren características particulares. Como afirman Silva y Baldissera "si es verdad que el poder no está dado de una vez por todas, también es verdad que los capitales instituidos (comunicados y reconocidos) se ejercen en perspectiva de conformar las relaciones de fuerza que los sujetos establecen al comunicarse" (2018, p.53, traducción propia).

Por otro lado, las circunstancias que han estimulado la desconfianza de una buena parte de las/los encuestadas/os (40\%) podrían estar afectando negativamente su sentido de corresponsabilidad y minando su interés por participar activamente para garantizar la buena marcha organizacional, participación que podría llevar a la priorización y consecución del interés común. A lo anterior se suman las percepciones de que las comunicaciones oficiales podrían no ser verdaderas.

Como planteó el MST desde su nacimiento en asentamientos y su desarrollo de cooperativas agrícolas "la participación verdadera se conquista con descentralización de informaciones, organización de los espacios de gestión (quién decide sobre qué y dónde), transparencia en las relaciones y persistencia en la práctica colectiva de participación" (Caldart, 1994, p.15, traducción propia).

Entendiendo que lo que sucede en las organizaciones debe analizarse en el contexto más amplio de los factores históricos, políticos, económicos y culturales, sus interacciones y tensiones; la interpretación de los datos arrojados por las investigaciones puede ser realizada a partir de una lectura contextual. Las interacciones entre el entorno macro y la vida organizacional no son en ninguna medida relaciones de causa-efecto, tampoco unidimensionales y mucho menos homogéneas. Siguiendo esa premisa, analizamos las características que adquieren la confianza y la vigilancia en las cooperativas cafetaleras costarricense, a partir de datos más generales de la confianza de las y los costarricenses.

Sin embargo, es posible ampliar el foco y analizar las dinámicas organizacionales, locales y nacionales en la perspectiva más amplia de lo regional. Concordamos con Henriques y Silva $(2018$, p.59) cuando apropiando la reflexión de Rosanvallon 
argumentan que "hay una fractura generalizada de la confianza de los sujetos en aspectos científicos, económicos, con el mundo cada vez más caracterizado por la imprevisibilidad financiera y por la especulación, y aspectos sociopolíticos, en especial acerca de la corrupción en instituciones y partidos políticos." En nuestros análisis de las dinámicas organizacionales podemos incluir tal desconfianza generalizada considerando los contextos de inestabilidad, inseguridad, individualismo y competencia exacerbadas, que caracterizaron las últimas décadas.

En la región latinoamericana, cuya brecha entre ricos y pobres sigue creciendo abrumadoramente y considerando que con la actual pandemia por el covid-19 las perspectivas futuras de este modelo de sociedad parecen ser aún más pesimistas; hoy más que nunca, las cooperativas son una alternativa (sin romanticismos ni purezas), precisamente por su interés prioritario por el bien común y por el papel histórico que han tenido y pueden seguir teniendo en la distribución equitativa de las riquezas.

En un contexto que promueve el egoísmo, la competencia y la ganancia individual, tanto como el desinterés por los asuntos comunes, colectivos y públicos; desestimulando con ello la participación ciudadana activa, comprometida y responsable, ser cooperativista es hacer contracultura. Este estudio pone en evidencia que, si deseamos fortalecer el sector, es urgente trabajar la comunicación con las personas asociadas, priorizando aquellas que todavía confían en el cooperativismo, en su filosofía y en su finalidad organizacional.

\section{REFERENCIAS}

ARAYA, Lisbeth Jiménez. Cooperativas cafetaleras costarricenses: características de la comunicación con sus asociados y asociadas. Mestrado: Universidad de Costa Rica, 2016. Disponível em: https://bit.ly/3g6W6lZ. Acesso em: 3 jun. 2021.

ARIZAGA, Cecilia. Sociología de la felicidad: autenticidad, bienestar y management del yo. Buenos Aires: Biblos, 2017.

BALDISSERA, Rudimar; BENETTI, Marcia (org.) Pesquisa e perspectivas de comunicação e informação. Porto Alegre: Sulina, 2018.

BAUMAN, Zygmunt. O mal-estar da pós-modernidade. Rio de Janeiro: Zahar, 1998.

BROWN, Wendy. Undoing the demos: neoliberalism's stealth revolution. New York: MIT, 2015.

CALDART, Roseli (ed.). Escola, trabalho e cooperação. movimento dos trabalhadores rurais sem terra, MST. Boletim da Educação, São Paulo, n. 4, 1994. Disponível em: https://mst.org.br/biblioteca-da-questao-agraria/ Acesso em: 15 jun. 2021.

COOPER, Melinda. Family values: between neoliberalism and the new social conservatism. New York: Zone, 2017.

CIRUELA, Antonio. La función directiva en las cooperativas agrarias: estudio empírico en las sociedades cooperativas cafetaleras de Costa Rica CIRIEC. Revista de Economía Pública, Social y Cooperativa, Valencia, v. 55, p. 253-288, 2006. Disponível em: https://www.redalyc.org/pdf/174/17405510.pdf. Acesso em: 3 jun. 2021.

EHRENBERG, Alain. O culto da performance: da aventura empreendedora à depressão nervosa. São Paulo: Ideias \& Letras, 2010. 
GARRIDO, Samuel. El cooperativismo agroalimentario en perspectiva histórica: ¿por qué no todos los agricultores cooperan? Mediterráneo Económico, Almeria, v. 24, p. 19-32, 2013. Disponível em: https://bit.ly/34KPXa0. Acesso em: 3 jun. 2021.

GAULEJAC, Vincent de. Gestão como doença social: ideologia, poder gerencialista e fragmentação social. São Paulo: Ideias\& Letras, 2017.

HENRIQUES, Márcio Simeone; SILVA, Daniel Reis. \#TáFaltandoÁgua: cidadania, vigilância civil e a produção de dados independentes. Revista Animus, Santa Maria, v. 17, n. 35, p. 56-74, 2018. doi: https://doi.org/10.5902/2175497724818.

HUERTAS HERNÁNDEZ, Olga Lúcia. La participación en una cooperativa rural de ahorro y crédito: un análisis desde la cultura organizacional. Cuadernos de Desarrollo Rural, [S. l.], v. 2, n. 55, 2005. Disponível em: https://revistas.javeriana.edu.co/ index.php/desarrolloRural/article/view/1245. Acesso em: 3 jun. 2021.

INEC. ENAHO. 2019. Nivel de pobreza por LP según características de los hogares y las personas, julio 2018 y julio $2019,2019$. Disponível em: https://bit.ly/3vQSifj. Acesso em: 31 jul. 2020.

INEC. ENAHO. 2019. Coeficiente de Gini por hogar y per cápita, julio 2010-2019. 2019. Disponível em: https://bit.ly/2S8rbh5. Acesso em: 31 jul. 2020.

MADRIGAL-PANA, Johnny. La confianza de los consumidores: Balance de tres administraciones (2002-2006, 2006-2010, 2010-2014), 2014. Disponível em: https://bit.ly/3cfqrxy. Acesso em: 3 jun. 2021.

MADRIGAL-PANA, Johnny. La confianza de los consumidores: Encuesta N.66.Mayo 2020. Disponível em: http://www.estadistica. ucr.ac.cr. Acesso em: 28 ago. 2020.

MARCHIORI, Marlene. Comunicación interna: una visión más amplia en el contexto de las organizaciones. Ciencias de la Información, v. 42, n.2, p. 49-54, 2011. Disponível em: https://bit.ly/3z13fNd. Acesso em: 3 jun. 2021.

MARTÍN-BARÓ, Ignacio. Sistema, grupo y poder: psicología social desde Centroamérica (II). 4. ed. El Salvador: UCA, 1999.

MBEMBE, Achille. Necropolítica: biopoder, soberania, estado de exceção, política da morte. São Paulo: N-1, 2018.

MOZAS Moral, Adoración. La participación de los socios en las cooperativas agrarias: una aproximación empírica CIRIEC. Revista de Economía Pública, Social y Cooperativa, Valencia, v. 40, p. 165-193, 2002. Disponível em: https://www.redalyc.org/ articulo.oa?id=17404008. Acesso em: 3 jun. 2021.

XIII Congreso Nacional Cooperativo. Consejo Nacional de Cooperativas CONACOOP, 2014. Disponível em: https://bit.ly/2Rh8XJW. Acesso em: 3 jun. 2021.

SALGADO, Julia. Entre solitários e solidários: o empreendedor nos discursos da Folha de S. Paulo (1972-2011), 2016. (Doutorado em Comunicação). Escola de Comunicação, Universidade Federal do Rio de Janeiro, Rio de Janeiro, 2016.

SILVA, Cássia; BALDISSERA, Rudimar. Comunicação organizacional e gestão de equipes: ausculta, estratégia e relações de poder. In: BENETTI, Marcia; BALDISSERRA, Rudimar. Pesquisa e perspectivas de comunicação e informação. Porto Alegre: Sulina, 2018. 
SILVA, Daniel Reis. Resposta aos nossos Críticos: discursos de defesa organizacional, créditos de confiança e pretensões de solidariedade In: ENCONTRO DA ASSOCIAÇÃO NACIONAL DOS PROGRAMAS DE PÓS-GRADUAÇÃO EM COMUNICAÇÃO, 29., 2020, Campo Grande. Anais [...] Campo Grande: Compós, 2020. Disponível em: https://bit.ly/3g9lGqn. Acesso em: 18 out. 2020.

Artículo recibido el 05.09.2020 y aprobado el 28.04.2021 